University of Nebraska - Lincoln

DigitalCommons@University of Nebraska - Lincoln

Faculty Publications, Department of Physics and Astronomy

Research Papers in Physics and Astronomy

$7-1-2004$

\title{
Hysteresis of Granular FePt:Ag Films With Perpendicular Anisotropy
}

\author{
M. L. Yan \\ University of Nebraska-Lincoln, myan@unlserve.unl.edu \\ Ralph Skomski \\ University of Nebraska-Lincoln, rskomski2@unl.edu \\ Arti Kashyap \\ akashyap@Inmiit.ac.in \\ L. Gao \\ Sy-Hwang Liou \\ University of Nebraska-Lincoln, sliou@unl.edu \\ See next page for additional authors
}

Follow this and additional works at: https://digitalcommons.unl.edu/physicsfacpub

Part of the Physics Commons

Yan, M. L.; Skomski, Ralph; Kashyap, Arti; Gao, L.; Liou, Sy-Hwang; and Sellmyer, David J., "Hysteresis of Granular FePt:Ag Films With Perpendicular Anisotropy" (2004). Faculty Publications, Department of Physics and Astronomy. 33.

https://digitalcommons.unl.edu/physicsfacpub/33

This Article is brought to you for free and open access by the Research Papers in Physics and Astronomy at DigitalCommons@University of Nebraska - Lincoln. It has been accepted for inclusion in Faculty Publications, Department of Physics and Astronomy by an authorized administrator of DigitalCommons@University of Nebraska Lincoln. 
Authors

M. L. Yan, Ralph Skomski, Arti Kashyap, L. Gao, Sy-Hwang Liou, and David J. Sellmyer

This article is available at DigitalCommons@University of Nebraska - Lincoln: https://digitalcommons.unl.edu/ physicsfacpub/33 


\title{
Hysteresis of Granular FePt:Ag Films With Perpendicular Anisotropy
}

\author{
M. L. Yan, R. Skomski, A. Kashyap, L. Gao, S. H. Liou, and D. J. Sellmyer
}

\begin{abstract}
Intergranular interactions in nanostructured FePt:Ag thin films and their effect on magnetic hysteresis are investigated. The films, produced by multilayer deposition plus rapid thermal annealing, consist of FePt nanoparticles embedded in a silver matrix. They are investigated by magnetization measurements and magnetic force microscopy. Analytical model calculations, supported by micromagnetic simulations, are used to elucidate the relation between coercivity, hysteresis-loop slope, and spatial correlations during magnetization reversal. The analytical calculations yield simple expressions for the loop slope and the coercivity as a function of the intergranular exchange. Small intergranular exchange enhances the coercivity, but for strong exchange there is a self-energy cutoff, associated with the onset of cooperative reversal and preventing alpha from becoming negative.
\end{abstract}

Index Terms-Anisotropy, magnetic recording, magnetization reversal.

\section{INTRODUCTION}

$\mathbf{T}$ HE need for ever-increasing storage densities in magnetic recording has lead to an intensive search for magnetic thin films with perpendicular magnetic anisotropy. This refers, in particular, to materials with $\mathrm{FePt}$ or $\mathrm{CoPt}$ as the main magnetic phase. A key aspect of the magnetism of particulate thin films is the relation between intergranular interactions, the hysteresis-loop shape, and spatial correlations. The specific interest in spatial correlations is fueled by the envisaged high storage density of advanced recording media and by the necessity to control noise. Victora et al. [1] have investigated the role of exchange and magnetostatic interactions, focusing on intragrain and grain-boundary effects. The shape of the hysteresis loop of magnetostatically interacting particles has been considered in [2], and various practical implications of the hysteresis-loop slope have been discussed by Honda et al. [3].

In this paper, we focus on the question how intergranular interactions affect the hysteresis. Particular emphasis is on the loop-slope parameter $\alpha=\mathrm{d} M / \mathrm{d} H\left(H_{\mathrm{c}}\right)$, and its largely unexplored relation to the real-space spin structure. We use FePt:Ag composite thin films [4] as a starting point and use model calculations and simulations to provide a qualitative explanation of the observed trends. Since full-scale micromagnetic simulations covering length scales from about $1 \mathrm{~nm}$ (grain boundaries) to several micrometers (macroscopic domains) go beyond present

Manuscript received October 16, 2003. This work was supported by INSIC, NSF-MRSEC (DMR-0213808), ARO, NRI, and CMRA.

The authors are with the Department of Physics and Astronomy and Center for Materials Research and Analysis, University of Nebraska, Lincoln, NE 68588 USA (e-mail: myan@unlserve.unl.edu; rskomski@unlserve.unl.edu; sliou@unl.edu; dsellmyer@unl.edu).

Digital Object Identifier 10.1109/TMAG.2004.832168
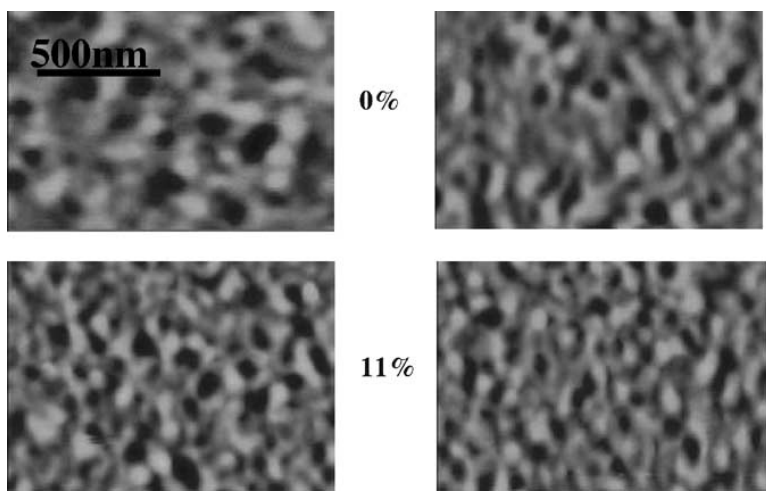

$11 \%$

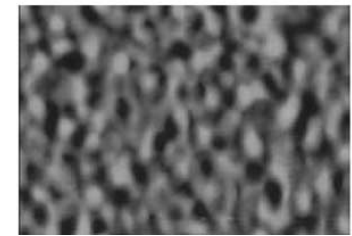

$20 \%$

Fig. 1. Magnetic-force micrographs of the thermally demagnetized FePt:Ag films for several Ag contents (vol\%).
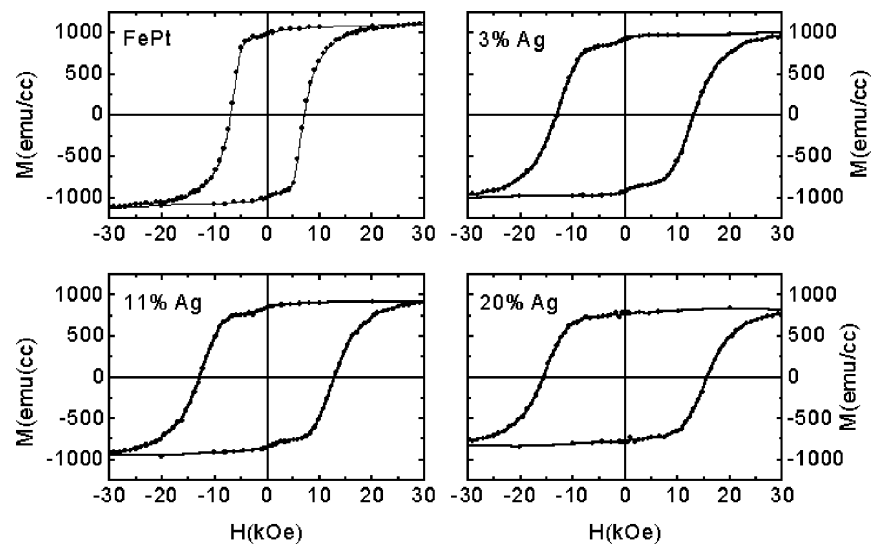

Fig. 2. Hysteresis loops for various Ag contents (vol\%).

numerical capabilities, we will use model calculations and simplified simulations.

\section{Structural And Magnetic Properties}

The granular films are produced by multilayer deposition plus rapid thermal annealing. Details of the processing and characterization of the films can be found in [4]. The films consist of FePt nanoparticles embedded in a silver matrix, and have varying particle sizes, packing fractions, and crystalline orientations [4]. Fig. 1 shows MFM micrographs of the films for several silver contents, which have been used to deduce average interaction-domain sizes (correlation lengths). Fig. 2 shows the hysteresis loops for the films; the loops have been used to determine the coercivities and the hysteresis-loop slope parameter $\alpha$.

Fig. 3 summarizes the magnetic properties of the films of Figs. 1 and 2. With increasing silver content the hysteresis-loop slope and the correlation length decrease, whereas the coercivity 


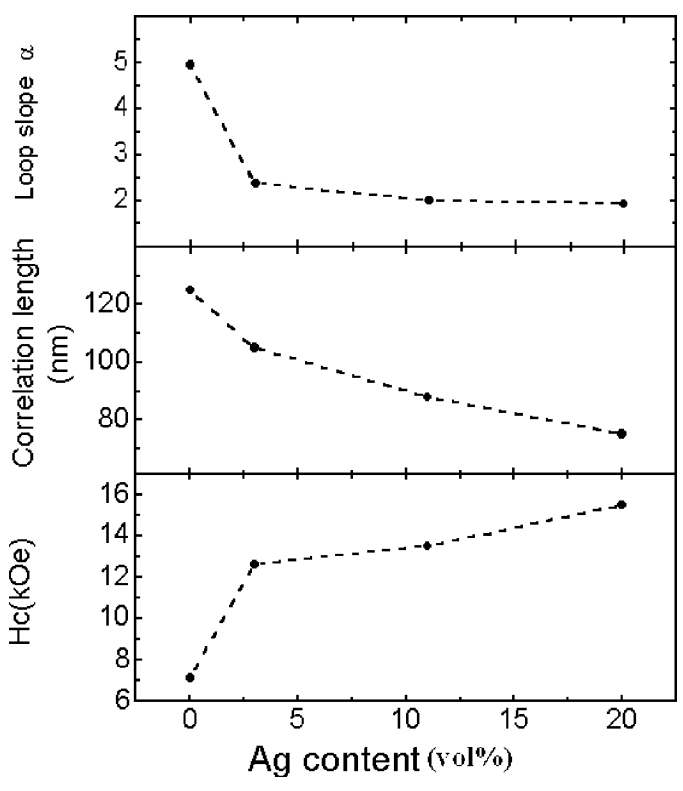

Fig. 3. Loop slope, correlation length, and coercivity for various Ag contents (vol\%).

increases. The silver increases the separation between the particles, thereby effectively exchange-decoupling the grains.

Figs. 1-3 reveal a close relationship between spatial and magnetic aspects of hysteresis. To explain this behavior, as well as similar features encountered in other particulate nanomagnets [2], [3], it is necessary to include the main physical properties of the grains, such as the single-grain hysteresis loops, and the magnetostatic and exchange interactions between grains. In terms of interaction fields, the magnetostatic and exchange interactions can be written as -DM and $\lambda \mathrm{M}$, respectively, where $\mathrm{D}$ is an effective demagnetizing factor and $\lambda$ is an exchange constant.

\section{HYSTERESIS-LOOP SLOPE}

By definition, the hysteresis-loop slope $\alpha=\mathrm{dM} / \mathrm{dH}\left(\mathrm{H}_{\mathrm{c}}\right)$ is a susceptibility: $\alpha=\chi\left(\mathrm{H}_{\mathrm{c}}\right)$. A convenient and quite general way of expressing the susceptibility of systems of interacting particles is to write

$$
\chi=\frac{\chi_{\mathrm{o}}}{1+\mathrm{cW}}
$$

where $\chi_{\mathrm{o}}$ is the interaction-free or single-grain susceptibility, $\mathrm{W}$ is the interaction strength, and $\mathrm{c}$ is a response parameter. Examples are the Curie-Weiss susceptibility $(\mathrm{W}=\mathrm{J})$ and the Stoner susceptibility $(\mathrm{W}=\mathrm{I})$. Physically, $1 / \chi=1 / \chi_{\mathrm{o}}+\mathrm{cW} / \chi_{\mathrm{o}}$ corresponds a sum of local and interparticle interaction fields.

Magnetostatic selfinteractions (the demagnetizing field) reduce $\alpha$. In a different context, this loop-slope modification is known as the skewing or 'shearing' correction of the hysteresis. Intergranular exchange has the opposite effect, so that (1) becomes

$$
\alpha=\frac{\alpha_{\mathrm{o}}}{1+\alpha_{\mathrm{o}}(D-\lambda)}
$$

where $\alpha_{\mathrm{o}} \approx M_{s} / \Delta H_{o}$ reflects the switching-field distribution $\Delta H_{\mathrm{O}}$ of noninteracting particles, which, in turn, depends on

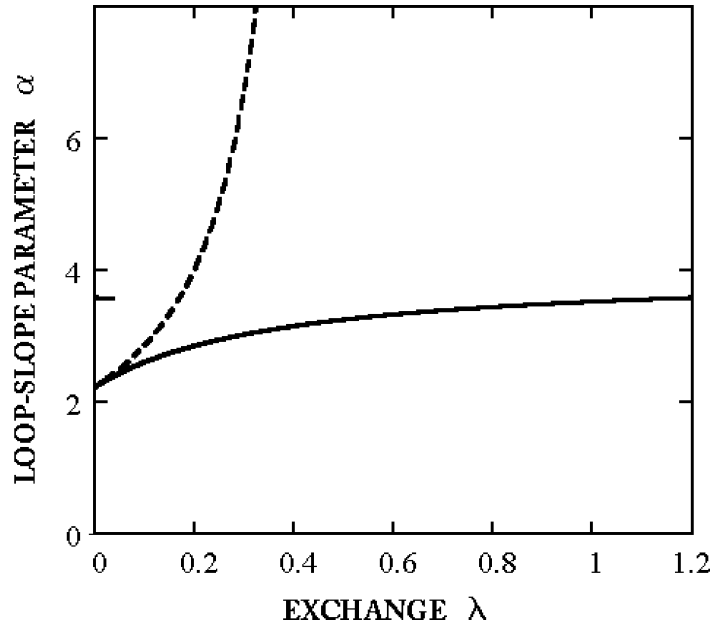

Fig. 4. Parameter $\alpha$ as a function of exchange interaction. The solid and dashed curves are predictions from (3) and (2), respectively. Since the dependence of the net exchange on the Ag content is unknown, no quantitative fitting has been attempted.

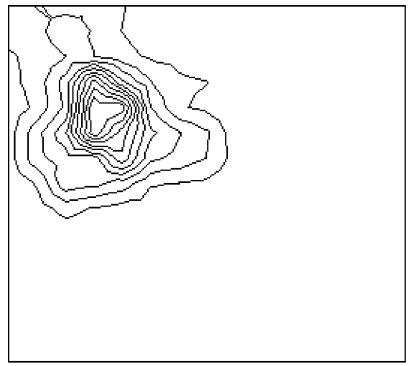

(a)

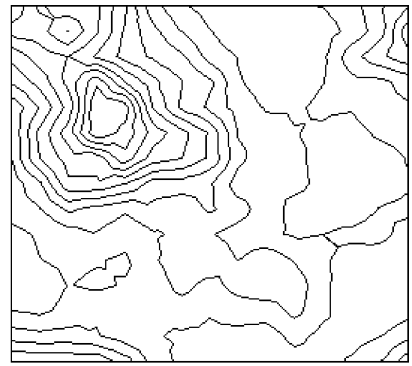

(b)
Fig. 5. Real-space interpretation of magnetization reversal: (a) weak exchange and (b) strong exchange. The figure shows the nucleation mode for $100 \times 100$ particles arranged on a square lattice. The mode is obtained by starting from a saturated state and then applying a reverse field until the saturated state becomes unstable.

real-structure features such as size distribution, particle shape, chemical disorder, and crystalline imperfections [5]. For example, narrow switching-field distributions correspond to large slopes. Fig. 4 shows a typical $\alpha(\lambda)$ curve.

The experimental decrease of $\alpha$ with increasing Ag content (Fig. 3) means that well-separated particles exhibit a very weak intergranular exchange parameter $\lambda$. Magnetostatic self-interaction has the opposite sign, because the demagnetizing factor of isolated spheres $(\mathrm{D}=1 / 3)$ is smaller than that of dense thin films $(\mathrm{D}=1)$.

\section{MEAN-Field AND COOPERATIVE EFFECTS}

The validity of (2) is restricted to the weak-interaction limit $\lambda-D<\Delta H_{o} / M_{s}$. Strong exchange leads to an unphysical overskewing of the loop, whereas strong demagnetizing fields lead to vortex and other flux-closure effects going beyond a simple demagnetizing field [5]-[7].

The main reason for this limitation is the mean-field character of $\lambda$ and D. It is well-known that mean-field theories work very well on a local scale but fail to account for long-range spatial correlations. Fig. 5 illustrates this point by showing nucleation modes for a square lattice of $100 \times 100$ particles with per- 
pendicular anisotropy. (For simplicity, the particle positions are not shown in the figure.) The particles exhibit some nonequivalence, modeled as anisotropy disorder $K_{1}\left(\mathbf{r}_{\mathbf{i}}\right) \neq\left\langle\mathrm{K}_{1}\right\rangle$, so that the nucleation of reverse domain is localized [7]. The localization length, which roughly corresponds to correlation length visible in Fig. 1, increases with increasing intergranular exchange, and for strong exchange, as in Fig. 5(b), the mean-field approach breaks down completely.

Another way of looking at this failure is to consider the onset of cooperative reversal. In the strong-exchange limit, that is, in dense films, $\lambda$ is essentially given by the molecular field. This field, at least a few 100 teslas, is much higher than any other involved field and indicates that (2) can no longer be used. Physically, strong interactions mean that two or more particles switch as a single unit, and any excess exchange has very little effect on the hysteresis loop. In other words, there is a self-energy cutoff that reduces the effect of very strong exchange on the hysteresis loop.

As shown for a two-particle model [7], cooperative effects amount to a narrowing of the switching-field distribution: The switching fields of individual particles are random quantities, and exchange interactions lead to an averaging of the switching fields over the localization length $\mathrm{L}$. In the numerical nucleation analysis of Fig. 5, this effect is automatically considered, but it can also be included in the model of (1) and (2). Restricting the consideration to two dimensions and exploiting well-known random-field arguments [8], [9] reveals that, for large $\lambda, L \sim \lambda$ and $\Delta H \sim 1 / \lambda$. The expression

$$
\alpha=\frac{\alpha_{\mathrm{o}}}{\alpha_{\mathrm{o}} D+\frac{1}{1+\alpha_{\mathrm{o}} \lambda}}
$$

realizes the above-mentioned cutoff and interpolates between the weak-coupling and strong-coupling regimes.

\section{DISCUSSION AND CONCLUSIONS}

Eqs. (2) and (3) predict that $\alpha$ approaches a finite value in the small-exchange limit of well-separated particles, in agreement with experiment (Fig. 3). Similarly, the decrease of the correlation length in Fig. 3 is explained in terms of the exchange-dependent correlation length visible in Fig. 5 . The relation between the correlation length and the coercivity is less straightforward. In nearly perfect magnets, the coercivity is nucleation-controlled and decreases with decreasing correlation length [7]. However, it can be shown that the opposite is true for strongly disordered magnets, whose coercivity is determined by domain-wall pinning. In this explanation, the relatively small drop in $\mathrm{H}_{\mathrm{c}}$ for small Ag contents (bottom of Fig. 3) means that continuous films contain less pinning centers.

From a practical point of view, large values of $\alpha$ are unfavorable for high-density magnetic recording. This is because $D \approx 1$ for thin films and strong exchange tends to enhance the size of the cooperatively switching regions and therefore to reduce the storage density. However, there is no straightforward relationship between the hysteresis-loop shape and the exchange interaction. For example, ensembles of identical noninteracting Stoner-Wohlfarth particles exhibit $\alpha=8$, in spite of the absence of exchange.

In conclusion, our analytical model calculations, supported by micromagnetic simulations, explain the close relationship between coercivity, loop shape, and spatial correlation length. The analytical calculations yield simple expressions for the loop slope and the coercivity as a function of the intergranular exchange. For strong exchange there is a self-energy cutoff, associated with the onset of cooperative reversal and preventing $\alpha$ from becoming negative.

\section{REFERENCES}

[1] R. H. Victora, J.-H. Xue, and M. Patwari, "Areal density limits for perpendicular magnetic recording," IEEE Trans. Magn., vol. 38, pp. $1886-1891,2002$.

[2] T. Suzuki, H. Muraoka, Y. Nakamura, and K. Ouchi, "Design and recording properties of Fe-Pt perpendicular media," IEEE Trans. Magn., vol. 39, pp. 691-696, 2003.

[3] N. Honda, K. Ouchi, and S.-I. Iwasaki, "Desin consideration of ultrahigh-density magnetic recording media," IEEE Trans. Magn., vol. 38, pp. 1615-1621, 2002.

[4] Y. Shao, M. L. Yan, and D. J. Sellmyer, "Effects of rapid thermal annealing on nanostructure, texture and magnetic properties of granular FePt:Ag films for perpendicular recording," J. Appl. Phys., vol. 93, pp. 8152-8154, 2003.

[5] R. Skomski, "Nanomagnetics," J. Phys.: Condens. Matter, vol. 15, pp. R841-R896, 2003.

[6] R. Skomski, J.-P. Liu, and D. J. Sellmyer, "Quasicoherent nucleation mode in two-phase nanomagnets," Phys. Rev. B, vol. 60, pp. 7359-7365, 1999.

[7] R. Skomski and D. J. Sellmyer, "Cooperative magnetism and the Preisach model," J. Appl. Phys., vol. 89, pp. 7263-7265, 2001.

[8] E. Callen, Y. J. Liu, and J. R. Cullen, "Initial magnetization, remanence, and coercivity of the random anisotropy amorphous ferromagnet," Phys. Rev, vol. 16, pp. 263-270, 1977.

[9] Y. Imry and S.-K. Ma, "Random-field instability of the ordered state of continuous symmetry," Phys. Rev. Lett., vol. 35, pp. 1399-1401, 1975. 